\title{
A Major Allergen of Lymphatic Filarial Nematodes Is a Parasite Homolog of the $\gamma$-Glutamyl Transpeptidase
}

\author{
Edgar Lobos,* Regina Zahn,* Niklaus Weiss,* \\ and Thomas B. Nutman ${ }^{\dagger}$ \\ *Department of Medical Parasitology, Swiss Tropical Institute, \\ Basel, Switzerland \\ 'Laboratory of Parasitic Diseases, National Institute of Allergy and \\ Infectious Diseases, National Institutes of Health, Bethesda, \\ Maryland, U.S.A.
}

\begin{abstract}
Background: Bm2325, a major IgE-inducing antigen of the filarial parasite Brugia malayi has been implicated in the pathology of tropical pulmonary eosinophilia (TPE), a pulmonary syndrome thought to result from hypersensitivity to microfilariae.

Materials and Methods: Affinity-purified IgE to Bm2325 from patients with TPE was used to identify a complementary DNA (cDNA) from a $B$. malayi expression library. Sequence analysis of the cDNA revealed a hitherto unknown parasite protein. Immunoblotting of the recombinant filarial protein using sera of patients with TPE determined its IgE-binding capacity. Reactivity to human lung epithelial cell proteins was analyzed using murine anti-Bm2325 antibodies and serum from patients with TPE.

Results: The predicted protein is a homolog of the entire
\end{abstract}

precursor of the $\gamma$-glutamyl transpeptidase $(\gamma$-GT), a key enzyme in the synthesis and degradation of glutathione. The filarial precursor encodes both the heavy $(\mathrm{H})$ and the light $(\mathrm{L})$ chain subunits and shares structural similarities with the mammalian enzymes. The $\mathrm{Bm} 2325$ allergen was identified as the homolog of the enzyme light chain subunit. Murine antibodies against the recombinant parasite $\gamma$-GT cross-reacted with the human enzyme present in human airway epithelial cells, and human $\gamma$-GT is a target of antibodies present in the serum of patients with TPE.

Conclusion: Molecular mimicry between the parasite $\gamma$-GT homolog and the host membrane-bound $\gamma$-GT present in lung epithelial cells likely contributes to the pathogenesis observed in tropical pulmonary eosinophilia.

\section{INTRODUCTION}

Tropical pulmonary eosinophilia (TPE) is caused by an immunological hyperresponsiveness to the lymphatic filarial parasites Wuchereria bancrofti or Brugia malayi. This interstitial lung disease is part of the wide range of clinical manifestations that occur in people infected with lymphatic filarial parasites; those with TPE contrast markedly with the majority of individuals with filarial infection, who show minimal clinical reactions $(1,2)$. TPE occurs in many filarial endemic areas, including India, Southeast Asia, and Brazil. Acute TPE is a

Address correspondence and reprint requests to: Edgar Lobos, Swiss Tropical Institute, Socinstrasse 57, 4002 Basel, Switzerland. syndrome characterized by pulmonary and systemic signs and symptoms associated with highgrade eosinophilia $\left(>3000 / \mathrm{mm}^{3}\right)$ and extremely high levels of serum IgE and antifilarial antibodies $(2-5)$.

With TPE there is characteristically an absence of microfilariae in the blood. The pathogenesis of the TPE syndrome is thought to be initiated by lung parenchymal inflammation in patients highly sensitized to the filarial parasite. Microfilariae (MF) released into the circulation by the adult lymphatic filariae are opsonized by antifilarial antibodies and then cleared in the pulmonary vasculature. These trapped MF degenerate, releasing antigenic/allergenic compo- 
nents that trigger a local inflammatory process $(3,4)$ with accumulation and activation of eosinophils in the lungs (6).

Previous studies demonstrated a striking elevation of total and filarial-specific IgE in the bronchoalveolar lavage fluid (BAL) of patients with TPE along with high levels of parasite-specific IgG. Antifilarial IgE and IgG antibodies localized in the lower respiratory tract-lining fluid analyzed by Western blotting recognized only a subset of the filarial antigens detected by serum antibodies (7). The rapid fall of these antibodies localized to the lung (without a concomitant drop in serum antibody levels) observed in individuals with acute TPE treated with diethylcarbamazine (DEC) paralleled their clinical improvement. These findings argue for the involvement of these local antibodies in the pathogenesis of the syndrome, although the precise mechanisms underlying the initiation, maintenance, or disease progression in TPE remain unknown. How and which parasites antigens are involved directly or indirectly in the regulation of specific IgE synthesis also remains to be elucidated.

We have previously reported the biochemical and immunological characterization of Bm2325, a major IgE-inducing filarial antigen of Brugia malayi (8). Although Bm2325 was only a minor component of the parasite antigenic extract, it was shown to be an important target of specific immune responses in patients infected with $W$. bancrofti exhibiting TPE. The Bm2325 allergen was shown to be glycosylated and expressed in the MF and in adult worms; it induced specific lymphocyte proliferation and IgE antibody production in vitro from peripheral blood mononuclear cells (PBMC) derived from patients with either TPE or other filarial syndromes. The allergen was also involved in local immune responses, as anti-Bm2325 IgE antibodies were identified in the lungs of patients with TPE.

In this study, we report the isolation, molecular characterization, and high-level expression of an IgE-binding cDNA encoding Bm2325. Analysis of the derived amino acid sequence revealed that the cloned gene encodes a protein that is homologous to the enzyme $\gamma$-glutamyl transpeptidase $(\gamma$-GT) (E.C.2.3.2.2.). The reaction catalyzed by $\gamma$-GT is of major importance in the $\gamma$-glutamyl cycle, a metabolic pathway that accounts for the enzymatic synthesis and degradation of glutathione $(9,10)$. The $\gamma$-GT catalyzes the transfer of the $\gamma$-glutamyl moiety of glutathione (GSH) and of other $\gamma$-glutamyl compounds to various acceptors, mainly amino acids (cysteine, methionine, glutamine). This reaction, which initiates the extracellular utilization of $\mathrm{GSH}$, is involved in the crucial recovery of cysteine moieties. The enzyme also plays a role in the transport of amino acids and in the metabolism of leukotrienes $(9,10)$.

All mammalian $\gamma$-GT enzymes characterized so far are integral membrane proteins composed of two nonidentical subunits, the heavy $(\mathrm{H})$ and the light (L) chain, posttranslationally cleaved from a common precursor. The enzymes are located on a number of mammalian cells, particularly high concentrations being found on the apical membranes of epithelial cells. This is the first report and analysis of a cloned $\gamma$-GT homolog from a parasite and its identification as a major allergenic protein in nematodes. We demonstrate, also, the presence of autoantibodies to human $\gamma$-GT in the serum of patients with TPE. Understanding of the immune system's fine interaction with epitopes on the filarial $\gamma$-GT homolog at the cellular and humoral levels, as well as its possible genetic restriction, may provide novel insights into the pathogenesis of the TPE syndrome.

\section{MATERIALS AND METHODS}

\section{Parasites}

B. malayi adult worms were obtained from the TRS laboratories (Athens, GA, U.S.A.). Microfilariae (MF) were recovered from the blood of infected jirds (Meriones unguiculatus) obtained by puncture of the retro-orbital plexus. The MF were isolated from contaminating tissue by differential centrifugation on Percoll gradient centrifugation (11).

\section{Patient Population}

Individuals ( $n=7)$ with acute TPE were residents of areas surrounding Madras, India, or Recife, Brazil, both regions being endemic for bancroftian filariasis. The diagnosis of TPE was established according to standard criteria $(2,4)$. Europeans with no relevant history of exposure to filarial diseases or any helminth parasites were used as controls.

\section{Affinity Purification of Anti-Bm2325 IgE Antibodies}

Solubilized B. malayi antigen from adult worms was separated by SDS-PAGE according to a mod- 
ified procedure of Laemmli (12), on $12.5 \%$ preparative gels. Antigen was electrophoretically transferred to nitrocellulose (NC) as described (13). The region of interest $(23-25 \mathrm{kD})$ was cut out and the strips saturated with $2.5 \%$ bovine serum albumin (BSA) in wash buffer $(50 \mathrm{mM}$ Tris- $\mathrm{HCl}, \mathrm{pH} 8.0,150 \mathrm{mM} \mathrm{NaCl}, 0.3 \%$ Tween 20 , $0.05 \%$ Triton $\mathrm{X}-100)$. Affinity purification of anti-Bm2325 IgE antibodies was carried out by incubating the strips containing blotted Bm2325 with a serum pool of patients with TPE (final dilution, 1:30), previously preadsorbed of IgG antibodies by incubation with protein G Sepharose (Pharmacia Fine Chemicals, Piscataway, NY, U.S.A.) as described (8).

\section{Screening, cDNA Isolation, and Sequence Analysis}

A $\lambda$ gt 11 cDNA expression library prepared from mRNA of male B. malayi (kindly provided by Dr. M. T. Philipp, Tulane Regional Primate Research Center, U.S.A.) was screened with the affinity-purified anti-Bm2325 IgE antibodies and alkaline phosphatase-conjugated goat immunoglobulins to human IgE (Kirkegaard and Perry, Gaithersburg, MD, U.S.A.). Clones reacting positively were plaque purified and used to infect Escherichia coli strain Y1090. After induction with isopropyl- $\beta$-D-thiogalactoside (IPTG), the recombinant antigen was analyzed as described (14). The cDNA insert was isolated and subcloned into M13 mpl 8 and M1 3 mpl 9 vectors using SacI and PstI restriction sites and sequenced in from both directions using standard M13 forward and reverse primers. In addition, Bm2325 cDNA PstI/ HindIII, HindIII and SacI/HindII restriction fragments corresponding to the nucleotide sequences 1-777, 778-1131, and 1132-1939, respectively, were subcloned into appropriately digested $\mathrm{Ml} 3$ and sequenced by the dideoxynucleotide chaintermination method (15) using Sequenase (U.S. Biochemical, Cleveland, OH, U.S.A.) according to the manufacturer's protocol. The cDNA sequence was analyzed with the computer program of the University of Wisconsin Genetics Computer Group.

\section{Northern Blot Analysis}

The 1939-bp cDNA probe encoding the filarial $\gamma$-GT was radiolabeled with $\left[\alpha-{ }^{32} \mathrm{P}\right]$ dATP using a Random Prime Kit from Boehringer (Mannheim, Germany). Poly(A) ${ }^{+}$RNA from adult female worms was isolated by oligo (dT) cellulose chromatography (QIAGEN GmbH, Hilden, Germa- ny). One microgram of poly $(A)^{+}$RNA was dissolved in $10 \mu \mathrm{l}$ sterile water mixed with $2 \mu \mathrm{l}$ sterile $6 \times$ loading buffer, size-fractionated on a $1.4 \% 10 \mathrm{mM}$ Na-phosphate agarose gel. RNAs were electroblotted to a Nytran membrane (Schleicher and Schuell, Dassel, Germany) in 25 mM Na-phosphate buffer, pH 6.8. Northern blot analysis and washing conditions were performed as described (16).

\section{High-Level Expression of the Filarial Homologs of the $\gamma$-GT Precursor and of the Light Chain Subunit}

Two different constructs of the parasite $\gamma$-GT homolog were amplified by PCR, which included the precursor polypeptide termed $\gamma$-GT.1 (amino acids 25-590; see Figs. 1 and 2 below), using the primers 5'-GGGCCCGAATTCGATGAAGGGC-3' and $5^{\prime}$-CCCGGGGATCCCTAGTGATGGTGATGG TGATGATATCCTCCAGGATGC-3'. Another construct, homolog to the light chain (L) corresponding to amino acids 396-590 (see Figs. 1 and 2 below), was amplified using the primers $5^{\prime}$-GGG CCCGAATTCACATCACATGTTTCG- $3^{\prime}$ as the forward primer and the previously described reverse primer. The reverse primer encodes for six consecutive histidine residues at the $\mathrm{COOH}$ terminus of the proteins that facilitate affinity chromatography purification of full-length recombinant proteins through the high affinity of histidines for Ni-NTA (nickel-nitrilo-acetic acid) agarose. The purified PCR products were digested with EcoRI and BamHI and subcloned into the EcoRI/BamHI cleaved expression vector pJC20 under the control of phage T7 RNA polymerase promoter. The plasmid was kindly provided by Dr. J. Clos from the Bernhard Nocht Institute for Tropical Medicine, Hamburg, Germany (17). After transformation of $E$. coli $\mathrm{DH} 5 \alpha$ cells, the recombinant pJC20- $\gamma$-GT.l and pJC20-L plasmids were isolated and used to transform E. coli BL21 (DE3), which is lysogenic for the T7 RNA polymerase. Lysates of cells transfected with vector only were used as controls. IPTG-inducible expression of the parasite proteins typically represented $>20 \%$ of the total cellular proteins (not shown).

\section{Purification and Characterization of the Recombinant Filarial Homolog of the $\boldsymbol{\gamma}$-GT}

BL21 (DE3) cells transformed with either the pJC20- $\gamma$-GT.1 or the pJC20-L plasmids were grown in LB medium $(50 \mu \mathrm{g} / \mathrm{ml}$ ampicillin, $0.2 \%$ glucose) at $37^{\circ} \mathrm{C}$ until $\mathrm{A}_{600}=0.3$. The cultures 
were induced with $0.5 \mathrm{mM}$ IPTG and centrifuged $2 \mathrm{hr}$ later at $4000 \times g$ for $10 \mathrm{~min}$. After being washed with $0.85 \% \mathrm{NaCl}$, cells were resuspended in lysis buffer A $(6 \mathrm{M} \mathrm{Gu} \mathrm{HCl}, 0.1 \mathrm{M}$ Na-phosphate, $0.01 \mathrm{M}$ Tris- $\mathrm{HCl}, \mathrm{pH} 8.0$ ) for $\mathrm{l} \mathrm{hr}$ at room temperature, and the lysates were centrifuged at $50,000 \times g$ for $1 \mathrm{hr}$ at $4^{\circ} \mathrm{C}$. The supernatants containing the soluble filarial $\gamma$-GT.1 and the $\mathrm{L}$ subunit were incubated with $50 \%$ $\mathrm{Ni}$-NTA agarose for $\mathrm{l} \mathrm{hr}$ at room temperature. The recombinant filarial allergens were washed and eluted according to a standard protocol (QIAexpress System, QIAGEN). The collected fractions were analyzed by SDS-PAGE, pooled, and stepwise dialyzed for $24 \mathrm{hr}$ with buffer $\mathrm{C}$ (0.1 M Na-phosphate, $0.01 \mathrm{M}$ Tris- $\mathrm{HCl}, \mathrm{pH}$ 6.3, urea $8 \mathrm{M}$ down to $100 \mathrm{mM}$ ) and the protein concentration determined using BSA as standard (18). Two micrograms of each recombinant parasite protein run on SDS-PAGE were transferred to NC (13) and incubated overnight at $4^{\circ} \mathrm{C}$ with a serum pool of patients with TPE diluted 1:30. After washing, the membranes were incubated with conjugate as described above.

\section{Immunochemical Methods}

Antiserum was produced by injecting mice i.p. with $50 \mu \mathrm{g}$ of the filarial precursor $\gamma$-GT.l emulsified in $500 \mu \mathrm{l} 50 \%$ Freund's incomplete adjuvant for the first two immunizations and $25 \mu \mathrm{g}$ for the last boost. Titer and specificity of the antiserum were determined by Western blot analysis. Test sera were kept at $-20^{\circ} \mathrm{C}$ until used.

\section{Western Blot Analysis of Human Bronchial Epithelial Cells}

An aliquot of a primary cell culture of airway epithelial cells $\left(10^{6}\right.$ cells) derived from human bronchi isolated by thoracotomy of patients with lung cancer (kindly provided by Holger Klapproth, Department of Pharmacology, University of Mainz [19]) or human lung fibroblasts $\left(10^{6}\right.$ cells) (ECACC \#90020107) were subjected to SDS-PAGE performed under reducing conditions. The proteins ( $40 \mu \mathrm{g} / \mathrm{lane}$ ) were transferred to nitrocellulose filters. The blots were incubated for $\mathrm{l} \mathrm{hr}$ with mouse antisera raised against the recombinant precursor of the filarial polypeptide $(\gamma$-GT.1) at a 1:4000 dilution. Final incubation was performed for $1 \mathrm{hr}$ with alkaline phosphatase-conjugated goat anti-mouse IgG (Jackson Immuno Research, West Grove, PA, U.S.A.) diluted 1:5000 and developed as described (8).
Analysis of human bronchial epithelial cells was carried out using the serum pool from patients with TPE diluted 1:50 previously preabsorbed to blotted E. coli proteins $(3 \times)$. After overnight incubation at $4^{\circ} \mathrm{C}$, the blots were washed and incubated with goat anti-human IgG alkaline phosphatase conjugated second antibody diluted 1:5000 (Sigma Chemical Co., St. Louis, MO, U.S.A.) and visualized as described above.

\section{RESULTS}

\section{Isolation and Characterization of cDNA Clones}

Screening of approximately $6 \times 10^{5}$ recombinant phages with affinity-purified IgE antibodies to $\mathrm{Bm} 2325$ yielded two clones, designated $\mathrm{Bm} 3$ and $\mathrm{Bm} 3.1$, both containing an insert of $2.0 \mathrm{~kb}$. Restriction fragment analysis indicated that they coded for the same gene. To characterize the $\mathrm{Bm} 3$ clone further, lysates were prepared from infected cells (14) and analyzed by Western blot using sera from patients with TPE. Infection of cells with $\lambda$ gt 11 (with no cDNA insert) was used also as a control. The results indicated that the protein encoded by Bm3 had a striking IgE immunoreactivity (data not shown).

\section{Bm3 Nucleotide and Amino Acid Sequences}

The complete nucleotide sequence of the 1939bp-long Bm3 cDNA and its deduced amino acid sequence are shown in Fig. 1. The cDNA has a single long open reading frame of $1773 \mathrm{bp}$ encoding a protein of 590 amino acids with a calculated molecular weight of $65.44 \mathrm{kD}$ and an isoelectric point (pI) of 6.32. The $5^{\prime}$ and $3^{\prime}$ untranslated regions are 85 and $81 \mathrm{bp}$, respectively. The first methionine codon in the predicted open reading frame matches the consensus initiation sequences for translational start (20). The $3^{\prime}$ untranslated region is incomplete, since it contains neither the poly(A) tail nor the polyadenylation signal. According to the consensus pattern Asn-Xaa-Ser/Thr for protein glycosylation (21), the protein contains seven possible sites for $\mathrm{N}$-glycosylation (Fig. 1). Analysis of the $B$. malayi deduced amino acid sequence indicates that the protein may undergo other posttranslational modifications such as phosphorylation (1 cAMP-dependent phosphorylation site at position 580 and $10 \mathrm{Ck} 2$ phosphorylation sites) 


\footnotetext{
-85 TTGACACCAGACCAACTGGTAATGGTAGCGACCGGCGCTCAGCTGGAATTCCGGAACAATTGAAATCCGATATTGCATAATTGTT

1 ATGATAATGTTACTTAGGTTATTTATTATAGTGACTTTTCTCACTGAATTAATTCATGCCTTTCAAAAAATGGATGAAGGGCAATTACAT

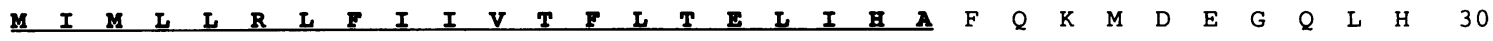

91 AagGAATATTTATGGCCACCACCTTCTGATAGTTATTTGGGAAAGTATATAAATGCAGCAGTTACCTGTGATCATGGTTTATGCAGTGAA $\begin{array}{llllllllllllllllllllllllllllllll}\mathrm{K} & \mathrm{E} & \mathrm{Y} & \mathrm{L} & \mathrm{W} & \mathrm{P} & \mathrm{P} & \mathrm{P} & \mathrm{S} & \mathrm{D} & \mathrm{S} & \mathrm{Y} & \mathrm{L} & \mathrm{G} & \mathrm{K} & \mathrm{Y} & \mathrm{I} & \mathrm{N} & \mathrm{A} & \mathrm{A} & \mathrm{V} & \mathrm{T} & \mathrm{C} & \mathrm{D} & \mathrm{H} & \mathrm{G} & \mathrm{L} & \mathrm{C} & \mathrm{S} & \mathrm{E} & 60\end{array}$

181 ATTGGCAGAAATATTTTGATCAGAGGTGGTAATGCAATTGATGCATCGATTGCTTCAATGTTTTGCCTTGGTATTACTAATCCTCAAAGT $\begin{array}{llllllllllllllllllllllllllllllllll}I & G & R & N & I & L & I & R & G & G & N & A & I & D & A & S & I & A & S & M & F & C & L & G & I & T & N & P & Q & S & 90\end{array}$

271 AGTGGAATTGGTGGTGGTTTTATTATGACTTTATATAATAGAAGTGAAGCAAAATGTACTGTTATTGATGCTAGAGAAACAGCACCTAAG $\begin{array}{lllllllllllllllllllllllllllllll}S & G & I & G & G & G & F & I & M & T & L & Y & \underline{N} & R & S & E & A & K & C & T & V & I & D & A & R & E & T & A & P & K & 120\end{array}$

361 AATGCTTCTCGAGATATGTTTATTAATGATGAATTTGGTTCTAAATATGGTTTTCGTGCTATAGCAACACCAGGTGAAATTGCTGGTTAT $\begin{array}{lllllllllllllllllllllllllllllll}\mathrm{N} & \mathrm{A} & \mathrm{S} & \mathrm{R} & \mathrm{D} & \mathrm{M} & \mathrm{F} & \mathrm{I} & \mathrm{N} & \mathrm{D} & \mathrm{E} & \mathrm{F} & \mathrm{G} & \mathrm{S} & \mathrm{K} & \mathrm{Y} & \mathrm{G} & \mathrm{F} & \mathrm{R} & \mathrm{A} & \mathrm{I} & \mathrm{A} & \mathrm{T} & \mathrm{P} & \mathrm{G} & \mathrm{E} & \mathrm{I} & \mathrm{A} & \mathrm{G} & \mathrm{Y} & 150\end{array}$

451 TGGCTTGCATTTAAACGCTTTGGTAGTGGTAAGATATCATGGTACGATCTGATAAAACCATCCATTGATTTATGTCGGTATGGGGTACCT $\begin{array}{lllllllllllllllllllllllllllllllll}\text { W } & \text { L } & \text { A } & \text { F } & \text { K } & \text { R } & \text { F } & \text { G } & \text { S } & \text { G } & \text { K } & \text { I } & \text { S } & \text { W } & \text { Y } & \text { D } & \text { L } & \text { I } & \text { K } & \text { P } & \text { S } & \text { I } & \text { D } & \text { L } & \text { C } & \text { R } & \text { Y } & \text { G } & \text { V } & \text { P } & 180\end{array}$

541 GTGTCAAAGTATCTTGGATATGTCCTTGGTGTTAAAGAGAAACATTTTAGGACATTACCTTCTATGAAAAGTTGGATTAATAATGCTACT $\begin{array}{llllllllllllllllllllllllllllllll}V & S & K & Y & L & G & Y & V & L & G & V & K & E & K & H & F & R & T & L & P & S & M & K & S & W & I & N & N & A & T & 210\end{array}$

631 GATAAAGTATTTGTTGCTGGTGATATTATCAAACGACCTGAATTAGGTAATACATTAGAAAAGCTTGCACTTAGTTCTGATCCAGTGGAA $\begin{array}{llllllllllllllllllllllllllllllll}\mathrm{D} & \mathrm{K} & \mathrm{V} & \mathrm{F} & \mathrm{V} & \mathrm{A} & \mathrm{G} & \mathrm{D} & \mathrm{I} & \mathrm{I} & \mathrm{K} & \mathrm{R} & \mathrm{P} & \mathrm{E} & \mathrm{L} & \mathrm{G} & \mathrm{N} & \mathrm{T} & \mathrm{L} & \mathrm{E} & \mathrm{K} & \mathrm{L} & \mathrm{A} & \mathrm{L} & \mathrm{S} & \mathrm{S} & \mathrm{D} & \mathrm{P} & \mathrm{V} & \mathrm{E} & 240\end{array}$

721 CTTTTCTATCGTGGTGATATAGCTAAAGCATTGGTCAAAGAAATTGAAAATAATGGTGGAATTATTACGCTGGATGATTTCGCGCAATTT $\begin{array}{llllllllllllllllllllllllllllllllll}\text { L } & F & \text { Y } & \text { R } & G & \text { D } & \text { I } & \text { A } & \text { K } & \text { A } & \text { L } & \text { V } & \text { K } & \text { E } & \text { I } & \text { E } & \text { N } & \text { N } & \text { G } & \text { G } & \text { I } & \text { I } & \text { T } & \text { L } & \text { D } & \text { D } & \text { F } & \text { A } & Q & \text { F } & 270\end{array}$

811 AAGCCAACAGTACATGAGGAACCATTGATCAATGATCACTTCTCAGGTGATCTAGCAATGTGTGGTCCACCACCACCTTCCTCATTTGCT $\begin{array}{lllllllllllllllllllllllllllllllll}K & P & T & V & H & E & E & P & \text { L } & \text { I } & \text { N } & \text { D } & \text { H } & \text { F } & \text { S } & \text { G } & \text { D } & \text { L } & \text { A } & \text { M } & \text { C } & G & \text { P } & \text { P } & \text { P } & \text { P } & \text { S } & \text { S } & \text { F } & \text { A } & 300\end{array}$

901 GTTACTCAGCTAATCATATCACTTATGGCTCGCTTTTATGGACCTAAAACAAGCAAAGAGGTATTAAAACGTGATCCACTCTTTTATCAT $\begin{array}{llllllllllllllllllllllllllllllllll}V & T & Q & L & I & I & S & L & M & A & R & F & Y & G & P & K & T & S & K & E & V & \text { L } & K & R & D & P & \text { L } & F & Y & H & 330\end{array}$

991 CGACTTTTGGAGGCACAAAAATTTGCATATGCGCAACGAACACTTATGGGTGATGAAGCTTTTGTGGAAGAAGCTAAAGAGCTTGCAAAG $\begin{array}{lllllllllllllllllllllllllllllllll}R & \text { L } & \text { L } & E & \text { A } & Q & \text { K } & \text { F } & \text { A } & \text { Y } & \text { A } & \text { Q } & \text { R } & \text { T } & \text { L } & \text { M } & \text { G } & \text { D } & \text { E } & \text { A } & \text { F } & \text { V } & \text { E } & \text { E } & \text { A } & \text { K } & \text { E } & \text { L } & \text { A } & \text { K } & 360\end{array}$

1081 AATATGACAACAAAAGCATACACAGATTGGATATTCTCGAGAATGCGCAATAAAACACAACCAACAGAATACTATGGTGAGCTTCAGAAA $\begin{array}{llllllllllllllllllllllllllllllllll}\mathbf{N} & M & \text { T } & T & K & A & Y & T & D & W & I & F & S & R & M & R & \underline{N} & K & T & Q & P & T & E & Y & Y & G & E & L & Q & K & 390\end{array}$

1171 CAGCTAAATGATCATGGTACATCACATGTTTCGGTATTAGATTCTATGGGTAATGGTGTATCAAGTACAAGTACTGTTAATCGATGGTTT $\begin{array}{llllllllllllllllllllllllllllllll} & \mathrm{L} & \mathrm{N} & \mathrm{D} & \mathrm{H} & \mathrm{G} & \mathrm{T} & \mathrm{T} & \mathrm{H} & \mathrm{V} & \mathrm{S} & \mathrm{V} & \mathrm{L} & \mathrm{D} & \mathrm{S} & \mathrm{M} & \mathrm{G} & \mathrm{N} & \mathrm{G} & \mathrm{V} & \mathrm{S} & \mathrm{S} & \mathrm{T} & \mathrm{S} & \mathrm{T} & \mathrm{V} & \mathrm{N} & \mathrm{R} & \mathrm{W} & \mathrm{F} & 420 \\ \end{array}$

1261 GGCGCAGTAGTGCAATCTGTTGATTTGGGTGTTGTTTTTAATGATGAAATGGATGATTTTTCTACGCCAGGAATGGCTAATGGTTTCGGA \begin{tabular}{llllllllllllllllllllllllllllllllll}
\hline$G$ & $A$ & $V$ & $V$ & $Q$ & S & V & D & L & G & V & V & F & N & D & E & M & D & D & F & S & T & P & G & M & A & N & G & F & G & 450
\end{tabular}

1351 TTTGCACCATCGGAAAGTAATTTTATTGAACCGGGCAAGAAACCAATGAGTAGTATGAGTCCAATGATTGTTTATAATACTAAAACTGGA $\begin{array}{llllllllllllllllllllllllllllllllll}F & A & P & S & E & S & N & F & I & E & P & G & K & K & P & M & S & S & M & S & P & M & I & V & Y & N & T & K & T & G & 480\end{array}$

1441 AAAATTGTAATGGTAGCTGGTGCATCTGGTGGTTCAAAAATAATTTCTGCTTTAGCTAAACCTATTATTCGAGTATTATTTTTCAATGAA $\begin{array}{llllllllllllllllllllllllllllllll}K & I & V & M & \text { V } & A & G & A & S & G & G & \text { S } & K & \text { I } & \text { I } & \text { S } & \text { A } & \text { L } & \text { A } & \text { K } & \text { P } & \text { I } & \text { I } & \text { R } & \text { V } & \text { L } & \text { F } & \text { F } & \underline{N} & \text { E } & 510\end{array}$

1531 ACAATAAAAGAAGCAATTGATGCGCCAACATTACACAATCAATTTACGCCAGATATTACTCAATTTGAACAAACTGTACCTAAGGAATTG

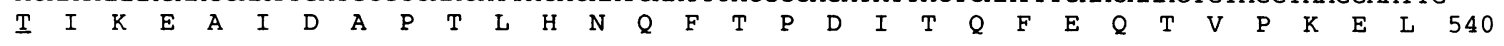

1621 ATGGATGATTTGACGCAAATTTTTGGACAAAAATTCAATAAAACCACTGGTTTTGAAGGTATAGCTCAAGGAATTGTTGTTGATGAGGAT $\begin{array}{llllllllllllllllllllllllllllllllllll}M & D & D & L & T & Q & I & F & G & Q & K & F & \underline{N} & K & T & T & G & F & E & G & I & A & Q & G & I & V & V & D & E & D & 570\end{array}$

1711 GGAACAATTTACGCCAATGGTGACTATAGGCGCCAAAGTGATATGCATCCTGGAGGATATTAGCAATAAAATTTTTTCAATTCATATATA $\begin{array}{llllllllllllllllllllll}\text { G } & T & I & Y & A & N & G & D & Y & R & R & Q & S & D & M & H & P & G & G & \text { Y } & & 590\end{array}$

1801 ATTATCCTCTAAATTTCCGCCGATACTGACGGGCTCCAGGAGTCGTCGCCACCA
}

FIG. 1. Nucleotide and derived amino acid sequence of the cDNA coding for the filarial homolog of the $\gamma$-GT

Numbers on the left indicate the nucleotide number in the open reading frame. Numbers on the right indicate the amino acid residue. The numbering of the amino acids starts at the initiation codon. The predicted signal peptide, residues 1-20 (boldface), and the putative N-glycosylation sites are underlined. The thick arrow shows the presumed cleavage site for the processing of the heavy and light chain subunits. The $\gamma$-GT signature pattern motif corresponding to the sequences previously published (31) is boxed. 


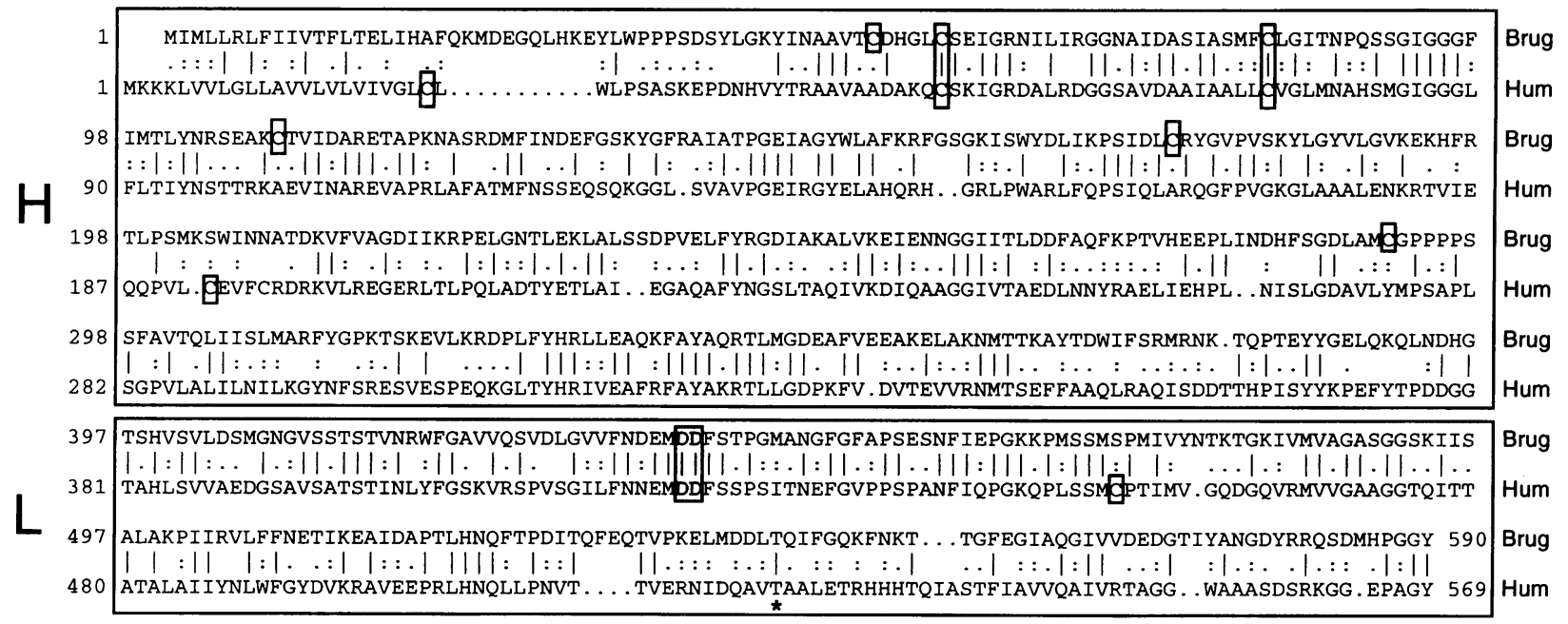

FIG. 2. Comparison of the B. malayi $\boldsymbol{\gamma}$-GT homolog and human $\boldsymbol{\gamma}$-GT-deduced amino acid sequences The amino acid sequence from the cDNA of $B$. malayi was aligned to that of human $\gamma$-GT (26). The H and L subunits are boxed. Gaps (indicated by dashes) have been introduced to maximize best pairing of homologous amino acids; numbers indicate the amino acid residue in the ORF. Identical residues are indicated by bars $(\mid)$; conservative substitutions by two points (:). The shaded box indicates the position of the two crucial Asp residues located in the active site of the enzyme. Shared or unique cysteine residues are boxed. The asterisk $\left({ }^{*}\right)$ indicates the position of Thr-555 corresponding to human Thr-524 involved in the binding of acivicin.

located at positions $110,143,163,263,273,317$, $401,511,530$, and 556 (22,23). Interestingly, a consensus sequence for glycosaminoglycan attachment (S-G-X-G) was found at position 9l (24). Furthermore, the cell attachment sequence R-G-D shown to play a role in cell adhesion was found at position 244 (25). The nucleotide sequence data reported in this paper are available in the GenBank database under the accession number UT49836. A search of protein sequence data bases for sequence similarity revealed that the amino acid sequence of the Bm2325 cDNA showed strong identity to published sequences of mammalian and prokaryotic $\gamma$-GT.

\section{Bm3 Encodes a Protein with Homology to the Human $\boldsymbol{\gamma}$-GT}

A homology search revealed strong similarity between the amino acid sequence derived from $B$. malayi and human $\gamma$-glutamyl transpeptidases (26). The mammalian enzyme is a heterodimeric glycoprotein consisting of two polypeptide chains of unequal sizes, the heavy $(\mathrm{H})$ and the light (L) chain subunits, synthesized as a single precursor polypeptide $(27,28)$. The degree of identity of the parasite protein to the human $\gamma$-GT are 35 and $43 \%$ for the heavy and light chain, respectively. When conservative amino acids substitutions where taken into account, the similarity to the human $\mathrm{H}$ and $\mathrm{L}$ subunits raised to 57 and $66 \%$, respectively. The parasite protein is larger ( 590 amino acids) than the human (569 amino acids). A comparison of the amino acid sequences of the $B$. malayi protein and the human enzyme is shown in Fig. 2. The molecular weights of the $B$. malayi unequal heavy and light chain subunits-as calculated from the cDNA sequence-are 44.51 and $20.94 \mathrm{kD}$, respectively. The estimated molecular weight for the L subunit is in good agreement with that of the enzymatically deglycosylated L subunit (termed Bm2325), as we previously demonstrated (8). The homology of the $B$. malayi protein to other $\gamma$-GTs from vertebrates is in the same range of similarity as to the human $\gamma$-GT (rat 35 and 43\%, pig 36 and $42 \%$ for the $\mathrm{H}$ and $\mathrm{L}$ chains, respectively) $(30,31)$. The bacterial $\gamma$-GT gene from $E$. coli is, as expected, less similar to the filarial protein $(30$ and $37 \%$, respectively) (31). At the $\mathrm{N}$ terminus of the mature $B$. malayi $\mathrm{L}$ chain, the signature pattern motif common to all described $\gamma$-glutamyl transpeptidases is found: TSHVSVLD SMGNGVSSTSTVNRWFG (Fig. 1, boxed area). This region matches the known consensus sequence perfectly: T-[STA]-H-x-[ST]-[LIVM]-x(4)G-[SN]-X-V-[STA]-X-T-X-T-[LIVM]-N-x(1,2)-(FY)-G (31). In addition, the distribution of the potential glycosylation sites in the deduced parasite sequence (five in the $\mathrm{H}$ and two in the $\mathrm{L}$ subunits) is similar to the glycosylation patterns of mammalian enzymes $(26,29,30)$. 


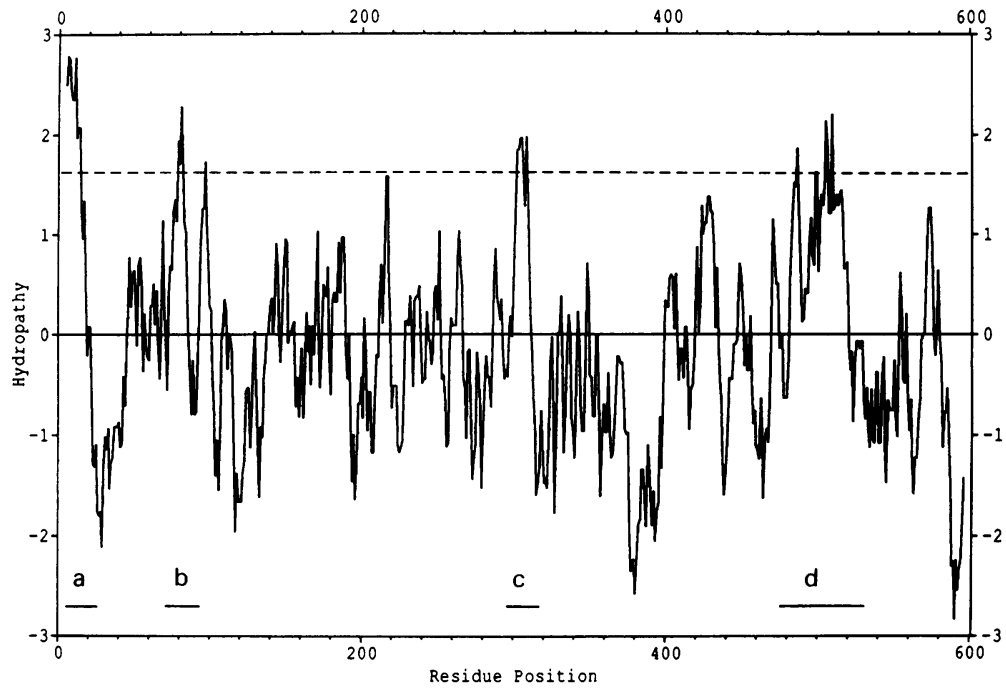

FIG. 3. Hydropathy plot analysis of the filarial $\gamma$-GT homolog

Hydrophobicity profile was obtained according to Kyte and Doolittle (32) using a search length of nine amino acids. Values for the hydrophobic and hydrophilic regions along the 590 amino acid sequence are above and below zero, respectively. The $\mathrm{N}$-terminal sequence is located at the left. The broken line corresponds to an average hydropathic index of 1.6. Hydrophobic sequences $\mathrm{A}, \mathrm{B}, \mathrm{C}$, and $\mathrm{D}$ with values above 1.6 correspond to similar regions of known $\gamma$-GTPs $(26,29,30)$.

\section{Hydropathy Analysis}

The deduced amino acid sequence derived from $B$. malayi was analyzed using the Kyte and Doolittle algorithm in a hydropathy plot (32), as shown in Fig. 3. The average hydrophobicity for a window of nine amino acids was plotted throughout the sequence. Analysis of the local hydrophobicity along the peptide revealed a strong similarity to hydrophobicity plots of known mammalian $\gamma$-GTs, showing three major hydrophobic regions (A, B, and C) with a hydropathy index greater than 1.6 in the $\mathrm{H}$ subunit and 1 (region D) in the L subunit $(26,29)$. Only region A ( 20 amino acids) is long enough to be a transmembrane domain.

\section{High-Level Expression and IgE Binding Capacity of the Recombinant Filarial $\boldsymbol{\gamma}$-GT Homolog}

Expression of the full-length precursor homolog of the $\gamma$-GT in a T7 RNA polymerase-dependent system (17) produced an IgE binding protein migrating with a molecular weight of $67 \mathrm{kD}$ (not shown); however, the yields obtained for the full-length polypeptide were extremely low. In contrast, much higher yields were obtained with construct $\gamma$-GT.1 (amino acids 25-590), which lacks the hydrophobic putative membrane-spanning domain A. After affinity chromatography purification on Ni-NTA agarose, the homologs of the precursor polypeptide ( $\gamma$-GT.1) and the mature L subunit (comprising amino acids 396590) migrated with an apparent molecular weight of 62 and 21-23 kD, respectively (Fig. 4, lanes 1 and 2). The recombinant products were subjected to Western blot analysis using a serum pool of patients with TPE and a serum pool of normal controls. Both recombinant proteins were shown to bind IgE strongly (Fig. 4, lanes 3 and 4). In addition, both recombinant antigens were recognized specifically by $\operatorname{IgE}$ antibodies present in the serum of individual patients with TPE (7/7) to varying extents (not shown). Therefore, IgE-binding epitopes were localized in the recombinant light chain subunit homolog previously characterized as the B. malayi Bm2325 allergen.

\section{Structural Aspects of the Filarial $\boldsymbol{\gamma}$-GT Homolog}

To investigate the structural nature of the filarial antigen, mouse antibodies raised against the purified recombinant $\gamma$-GT.l were used to analyze $B$. malayi adult protein extracts by immunoblot. Mouse antibodies against the polypeptide precursor recognized not only the homolog of the light (L) chain migrating with an estimated molecular weight of 23-25 kD but also the heavy chain $(\mathrm{H})$ subunit migrating with an apparent molecular weight of $48-58 \mathrm{kD}$ (Fig. 5, lane 2). In contrast, mouse preimmune serum showed no recognition of any protein at all (Fig. 5, lane 1). This finding indicates that, similar to prokaryotic and mammalian enzymes, the parasite $\gamma$-GT homologs are also heterodimeric proteins arising from a single polypeptide precursor $(31,33)$. 


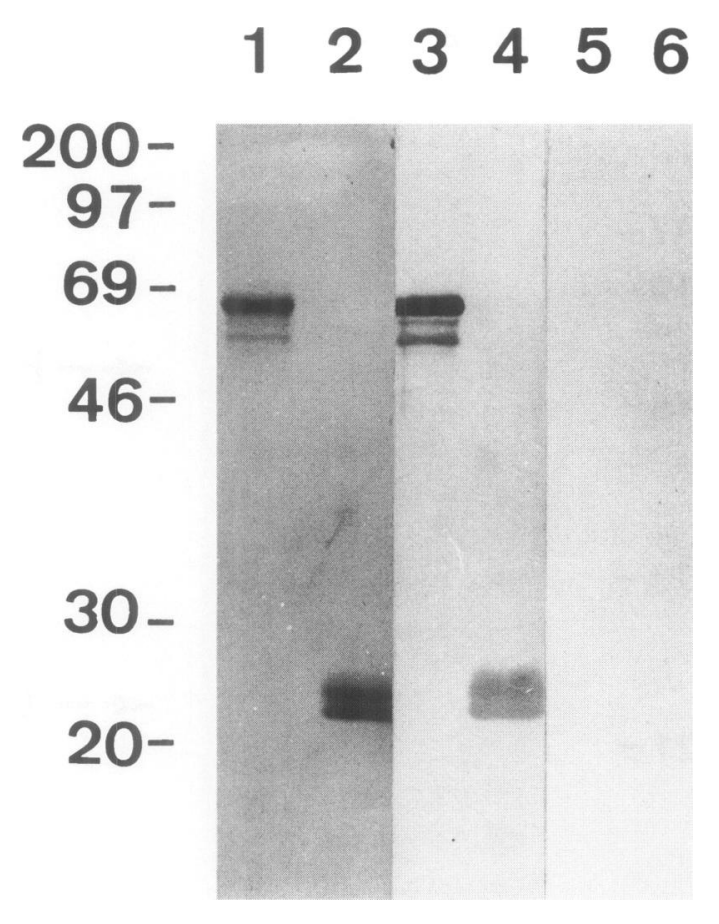

FIG. 4. Expression and Western blot analysis of the allergenicity of the recombinant $\gamma$-GT homolog

SDS-PAGE of recombinant proteins on $12.5 \%$ polyacrylamide gel stained with Coomassie blue of purified precursor polypeptide $\gamma$-GT.l (amino acids 25-590) or the mature light chain (L) homologs: Lanes 1 and 2, respectively; Lanes 3 and 4, immunoblot analysis showing the binding of human IgE antibodies from a serum pool of patients with TPE; Lanes 5 and 6, control reaction with a serum pool from normal individuals. Molecular size standards are indicated at the left.

\section{Analysis of Adult Filarial RNA}

To determine the size of mRNA coding for the filarial $\gamma$-GT homolog, the Bm3 cDNA was hybridized to a blot of poly $(\mathrm{A})^{+}$RNAs obtained from adult filarial worms. The detected filarial mRNA was approximately 2400 nucleotides long (Fig. 6).

\section{Immunological Crossreactivity between the Parasite Homolog and the Human $\gamma$-GT Enzyme, and Detection of Autoantibodies to the $\boldsymbol{\gamma}$-GT in Patients with TPE}

The extensive homologies found both at the structural and amino acid levels between the filarial $\gamma$-GT homolog and the human enzymeand the fact that $\gamma$-GTs are important membrane-bound enzymes of lung epithelial cellsprompted us to analyze the existence of immunologic cross-reactivity between them.

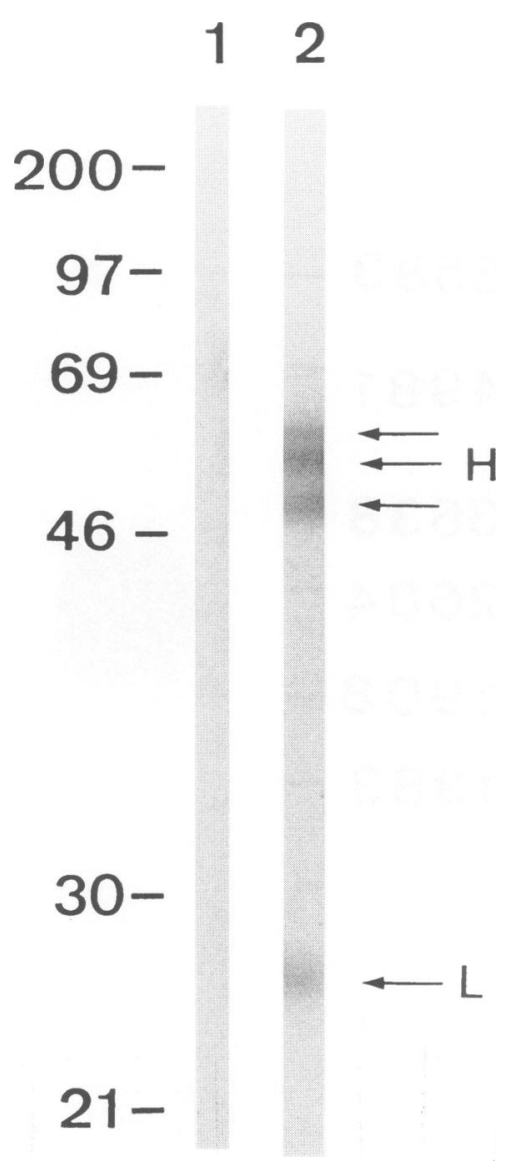

FIG. 5. Analysis of the filarial $\gamma$-GT homolog subunits by Western blot with mouse antibodies

B. malayi adult protein extract separated by denaturing SDS-PAGE on $12.5 \%$ gels was blotted as described (14). Lane 1, incubation with preimmune serum as a control; Lane 2, incubation with mouse anti- $B$. malayi $\gamma$-GT homolog antibodies. The arrows show the positions of the light chain subunit (L) (molecular weight 23-25 kD) (Bm2325) and the heavy chain subunit $(\mathrm{H})$ migrating with a molecular weight of $48-58 \mathrm{kD}$.

Mouse antibodies raised against the recombinant filarial $\gamma$-GT precursor homolog were used to probe protein extracts of human bronchial epithelial cells separated by SDS-PAGE under reducing conditions and electrophoretically transferred to nitrocellulose. Western blot analysis demonstrated that antiserum raised against the parasite precursor polypeptide recognized both $\mathrm{L}$ and $\mathrm{H}$ subunits of the human $\gamma$-GT present in the epithelial cells of human bronchi (shown in Fig. 7, Lane 1). In contrast, mouse preimmune serum used as control showed no recognition at all (Fig. 7, Lane 2). No specific immunoreactivity 


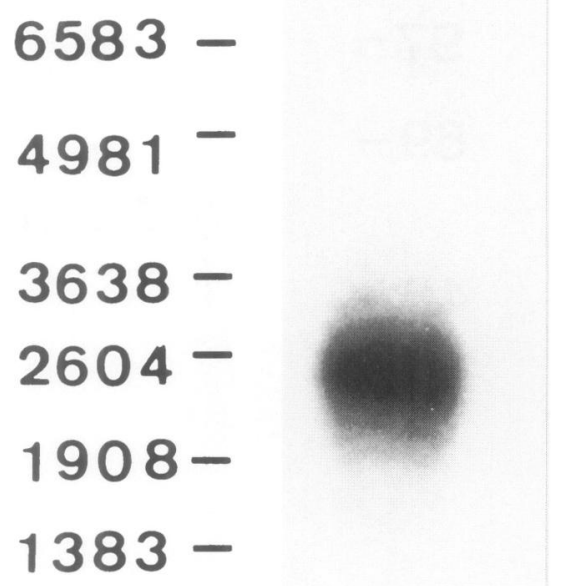

FIG. 6. Northern blot analysis and expression of the filarial $\boldsymbol{\gamma}$-GT homolog

Five micrograms of total RNA isolated from $B$. malayi adult females were electrophoresed, transferred, and hybridized to the $\mathrm{Bm} 3 \mathrm{cDNA}$ as a probe $\left(1 \times 10^{7}\right.$ cpm at a specific activity of $10^{8} \mathrm{cpm} / \mu \mathrm{g}$ ) according to the conditions previously described (16). The positions of RNA markers run in parallel are indicated at the left.

was observed using the same sera on blots of human lung fibroblasts used as control (Fig. 7, Lanes 3 and 4). It has been demonstrated that pulmonary tissues express not only the classical form of $\gamma$-GT but also unique $\gamma$-GT transcripts of unknown function that translate into a portion of the heavy chain and the entire light chain (34). The additional bands detected by the mouse hyperimmune serum might represent such shorter forms of the $\gamma$-GT or membrane-bound enzyme fragments. More important, antibodies present in the serum pool of patients with TPE recognized the $\gamma$-GT present in the human bronchial epithelial cells (Fig. 7, Lane 5). Control sera exhibited no immunoreactivity to the human epithelial cells (Fig. 7, Lane 6). The calculated molecular weight of 64 and 21-22 kD for the $\mathrm{H}$ and $\mathrm{L}$ subunits are in good agreement with the

\section{$\begin{array}{llllll}1 & 2 & 3 & 4 & 5 & 6\end{array}$}

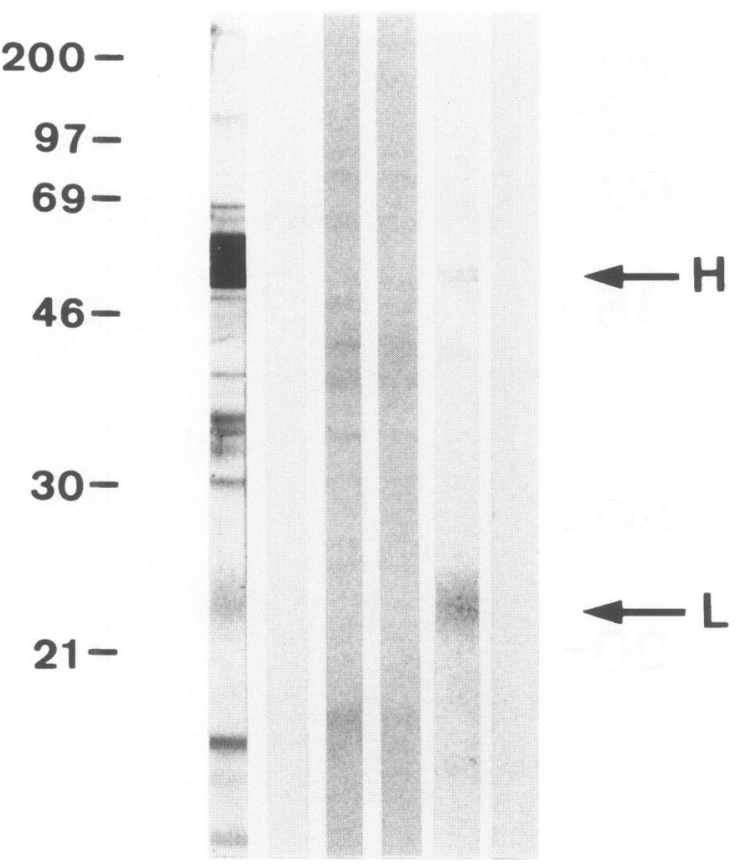

FIG. 7. Immunoreactivity of mouse anti-filarial $\gamma$-GT homolog antibodies and the serum patients with TPE toward human lung epithelial cells

Western blot analysis of cell extracts from primary cell cultures of lung epithelial cells or human lung fibroblasts used as control. The blots of lung epithelial cells were incubated with mouse antifilarial $\gamma$-GT homolog antiserum (Lane 1) and mouse preimmune serum (Lane 2); Lanes 3 and 4, blots of human lung fibroblasts incubated with mouse antifilarial $\gamma$-GT homolog antiserum or mouse preimmune serum, respectively; Lane 5, immunoreactivity toward human airway epithelial cells in the serum pool of patients with TPE; Lane 6 , reactivity of a serum pool of normal individuals. Arrows indicate the sizes of the human $\gamma$-GTs heavy $(\mathrm{H})$ and light subunits $(\mathrm{L})$.

estimated molecular weight for the human $\gamma$-GT subunits (33). Interestingly, these results also show the extremely fine specificity of the autoreactive antibodies induced in humans in the context of infection and of the pulmonary pathology observed as opposed to the mouse antibodies obtained in the context of hyperimmunization.

\section{DISCUSSION}

In this study, we report the identification of the allergen $\mathrm{Bm} 2325$ from the lymphatic filarial parasite $B$. malayi as the homolog of the membrane- 
bound enzyme $\gamma$-glutamyl transpeptidase, one of the key enzymes of the $\gamma$-glutamyl cycle. This finding is particularly relevant since human $\gamma$-GT is localized in lung epithelial cells and because of the important role that $\gamma$-GT plays in the antioxidant defense mechanism of several pulmonary cell types.

Immune responses to parasite $\gamma$-GT, involving IgE, IgG antibodies, and their effector cells, could thus lead to pathology in host lung tissues. Cross-reactivity between the parasite allergenic epitopes and self epitopes would then lead to autoreactivity due to molecular mimicry. In addition to the extensive homology between both sequences at the protein level, the $B$. malayi deduced amino acid sequence shares structural features with other eukaryotic $\gamma$-glutamyl transpeptidases, such as (i) synthesis as a single precursor polypeptide chain, (ii) a heterodimeric structure, (iii) similar hydropathy profiles, (iv) a large number of putative glycosylation sites resulting in multiple isoforms, and $(\mathrm{v})$ the presence of the sequence motif known as the $\gamma$-GT signature pattern.

The parasite sequence, however, has unique interesting structural features that make it different from the mammalian enzymes. The first 45 amino acids at the $\mathrm{N}$ terminus of the polypeptide precursor of the parasite's protein showed the weakest similarity to the host enzyme. In all mammalian $\gamma$-GTs, the signal peptide present in the precursor sequence is not cleaved and acts as the transmembrane-spanning domain (35). In $E$. coli, the $\mathrm{N}$-terminal signal peptide is cleaved and the mature enzyme is located in the periplasmic space without being anchored in the membrane (33). A putative signal peptide with a predicted cleavage at position 20 was found in the $B$. malayi sequence (36). Whether this cleavage site is used, or if the signal peptide remains in the membrane, is not known. Further biochemical experiments are in progress to clarify this issue.

Recently, $\gamma$-GT from the nematode Ascaris suum has been isolated and found to be predominantly associated with membranes (37). Interestingly, the sizes of the A. suum subunits (molecular weight of 43 and $30 \mathrm{kD}$ for the $\mathrm{H}$ and $\mathrm{L}$ subunits) differed from the mammalian subunits and did not show any size heterogeneity; differences between the filarial and Ascaris enzymes may therefore also exist. Western blot analysis of protein extracts from adult Ascaris worms using mouse antisera against the B. malayi $\gamma$-GT homolog identified both $\gamma$-GT subunits, giving evidence of immunological crossreactivity between both proteins (E. Lobos, unpublished observation). In contrast to the mammalian enzymes, the $\gamma$-GT from the protozoan Trypanosoma cruzi is cytosolic, is thermolabile above $30^{\circ} \mathrm{C}$, had different specificity to amino acid acceptors, and had different sensitivity to inhibitors (38). Such differences in substrate specificity and response to inhibitors suggests differences in the active sites of the parasites and the human enzymes.

Although the human $\gamma$-GT is predicted to contain four cysteines in the heavy chain, only two of these are conserved in the $B$. malayi sequence (cysteines 58 and 82). In addition, the parasite enzyme contains four additional cysteine residues (cysteines 53, 109, 175, and 291) not present in the mammalian enzymes. The unique cysteine residue of the human light chain (Cys-454), conserved among those mammalian $\gamma$-GTs whose primary sequences are known, is not present in the parasite sequence. Mutants of the human $\gamma$-GT involving the Cys- 454 residue are fully active, leading to the conclusion that this thiol is not required for catalysis. More important, replacement of Asp-423 and, to a lesser extent, Asp-422 by Ala or Glu led to a major loss of enzymatic activity, suggesting that both Asp residues play a crucial role in the active center of the enzyme (39). Both residues are found in the parasite sequence (Asp-438 and Asp-439). Another important residue previously shown to be located at or near the active site and involved in the binding of the inhibitor acivicin, which is conserved in the homolog B. malayi $\gamma$-GT sequence, is Thr-555, corresponding to Thr-524 in the human or Thr-523 in the rat $\gamma$-GTs. This threonine is involved in the binding of the inhibitor acivicin (40).

Cysteine protease family members from plants and arthropods have been shown to be potent allergens. Proteolytic enzymes produced by many helminth parasites for penetration and migration through host tissue have been proposed to induce Th2-type responses preferentially (41). Recently, a cysteine protease of the nematode Nippostrongylus brasiliensis was reported to stimulate IgE/IgGl antibody responses in rats, suggesting that IgE/IgGl responses are preferentially induced by some of the worm antigens but not by others (42). In this context, treatment of the $\gamma$-GT with dissociating agents results in extensive degradation of the large subunit $(\mathrm{H})$, because a latent protease activity of the light subunit (L) appears to be unmasked (43). Further analyses should clarify whether the filarial $\mathrm{L}$ subunit shows such protease activity and if 
it plays a role in the induction of specific $\operatorname{IgE}$ antibodies observed during natural infection.

A second group of helminth-derived molecules that might preferentially induce polyclonal and antigen-specific IgE and IgG4 antibodies were identified from the analysis of 24 recombinant filarial proteins as protease inhibitors (44). Recently, major grass and plant pollen allergens such as Lol pXI, Ole I have been shown to be members of a family of soybean trypsin inhibitor-related proteins (45). Thus, the results from these studies suggest that some nematode antigens (exhibiting certain biological properties such as protease activity or protease inhibitors) would be capable of inducing preferentially Th2-like responses in genetically predisposed individuals if presented to the immune system in the appropriate context and in the adequate amounts.

Characterization of the molecules involved in the induction of antigen-specific IgE antibodies is also of clinical importance, in view of the recent findings of the causal relationship between IgE and antigen-induced eosinophil accumulation in the lungs. Indeed, using a murine model of eosinophilic inflammation, administration of a nonanaphylactogenic murine anti-IgE antibody before antigen challenge neutralized serum IgE and inhibited the accumulation of eosinophils into the lungs along with the production of IL-4 and IL-5 (46).

Thus, central to this study is the identification of the IgE-inducing filarial antigen $\mathrm{Bm} 2325$ as a parasite homolog of the crucial epithelial brush border enzyme $\gamma$-glutamyl transpeptidase with potential to induce autoreactivity. This finding may have important consequences for the understanding of the precise mechanisms that underlie the pathogenesis of certain pulmonary eosinophilic syndromes. Because we have identified anti-Bm2325 IgE antibodies in the lower respiratory tract epithelial lining fluid (8) and because murine antibodies raised to the recombinant parasite $\gamma$-GT crossreacted with the enzyme present in human airway epithelial cells, a role for antibody-mediated pathogenesis in the syndrome is suggested.

The role of $\gamma$-GT as a part of the antioxidant defense mechanism of cells has been shown in several pulmonary cell types $(47,48)$ but awaits clarification in parasites. Remarkably, $\gamma$-GT levels are increased by oxidative stress in rat alveolar L2 epithelial cells (48). The concomitant increase in the levels of activity and expression of the $\gamma$-GT is part of the adaptation process to oxidative stress that enhances the use of extracellular glutathione via the $\gamma$-glutamyl cycle. Immune responses directed against the parasite $\gamma$-GT homolog in the lungs could subvert the host capacity to adapt to oxidative stress; upregulation of $\gamma$-GT levels on host lung epithelial cells would mean more target antigen for crossreacting antiparasite $\gamma$-GT antibodies. The subsequent epithelial damage with the ensuing neuronal hyperresponsiveness could have important consequences for the development of pulmonary inflammation.

The identification and expression of the filarial homolog to the mammalian $\gamma$-GTs with strong IgE binding capacity and as target of autoantibodies will allow basic studies on the mechanisms involved in the pathology of pulmonary eosinophilia. The complete primary structure of the filarial $\gamma$-GT presented in this study should facilitate the detailed analysis of $B$ and $T$ cell epitopes present on this allergen. These findings could provide the basis for the development of novel and specific therapeutic approaches for the treatment of pulmonary allergic diseases.

\section{ACKNOWLEDGMENTS}

We thank Dr. Holger Klapproth from the Department of Pharmacology, University of Mainz, Germany, for providing the human epithelial lung cells, and Drs. Caroline Suzuki and Thomas Bürgin from the Departments of Biochemistry and Cell Biology of the Biozentrum, University of Basel, Switzerland, for helpful discussions of this work. This research was supported by a grant from the Swiss National Science Foundation (NF 31-42495.94).

\section{REFERENCES}

1. Neva FA, Ottesen EA. (1978) Tropical (filarial) eosinophilia. N. Engl. J. Med. 298: 1129-1131.

2. Ottesen EA. (1984) Immunological aspects of lymphatic filariasis. Trans. $R$. Soc. Trop. Med. Hyg. 73(Suppl): 9-18.

3. Spry CJF, Kumaraswami V. (1982) Tropical eosinophilia. Semin. Hematol. 19: 101-115.

4. Ottesen EA, Nutman TB. (1992) Tropical pulmonary eosinophilia. Annu. Rev. Med. 43: 417-424.

5. Rom WN, Vijayan VK, Cornelius MJ, et al. (1990) Persistent lower respiratory tract inflammation associated with interstitial lung 
disease in patients with tropical pulmonary eosinophilia following conventional treatment with diethylcarbamazine. Am. Rev. Respir. Dis. 142: 1088-1092.

6. Pinkston $P$, Vijayan VK, Nutman TB, et al. (1987) Tropical pulmonary eosinophilia: Characterization of the lower respiratory tract inflammation and its response to therapy. J. Clin. Invest. 80: 216-225.

7. Nutman TB, Vijayan VK, Pinkston P, et al. (1989) Tropical pulmonary eosinophilia: Analysis of antifilarial antibody localized to the lung. J. Infect. Dis. 160: 1042-1050.

8. Lobos E, Ondo A, Ottesen EA, Nutman TB. (1992) Biochemical and immunological characterization of a major IgE-inducing filarial antigen of Brugia malayi and implications for the pathogenesis of tropical pulmonary eosinophilia. J. Immunol. 149: 3029-3034.

9. Meister A, Tate SS, Ross LL. (1976) Membrane bound $\gamma$-glutamyl transpeptidase. In: Martinosi A (ed). The Enzymes of the Biological Membranes. Plenum, New York. Vol. 3, pp. 315-347.

10. Meister A. (1989) Metabolism and function of glutathione. In: Dolphin D, Poulson R, Avramovic O (eds). Glutathione: Chemical, Biochemical and Medical Aspects. Wiley, New York, pp. 367-476.

11. Bardehle G, Klonisch T, Schott HH, Stirm S, Zahner H. (1987) Isolation of pure sheets of Litomosoides carinii microfilariae. Parasitol. Res. 74: 188-190.

12. Laemmli UK. (1970) Cleavage of structural proteins during the assembly of the head of bacteriophage T4. Nature 227: 680-685.

13. Towbin H, Staehelin T, Gordon J. (1979) Electrophoretic transfer of proteins from polyacrylamide gels to nitrocellulose sheets. Procedure and some applications. Proc. Natl. Acad. Sci. U.S.A. 76: 4350-4354.

14. Lobos E, Altmann M, Mengod G, Weiss N, Rudin W, Karam M. (1990) Identification of an Onchocerca volvulus cDNA encoding a lowmolecular-weight antigen uniquely recognized by onchocerciasis patients. Mol. Biochem. Parasitol. 39: 135-146.

15. Sanger F, Nicklen S, Coulson AR. (1977) DNA sequencing with chain-terminating inhibitors. Proc. Natl. Acad. Sci. U.S.A. 74: 54635467.

16. Pelle R, Murphy NB. (1993) Northern hybridization: Rapid and simple electrophoretic conditions. Nucleic Acids Res. 21: 2783-2784.

17. Clos J, Brandau S. (1994) pJC20 and
pJC40 - Two high copy-numbers vectors for T7 RNA polymerase-dependant expression of recombinant genes in Escherichia coli. Protein Exp. Purif. 5: 133-137.

18. Bradford MM. (1976) A rapid and sensitive method for the quantitation of microgram quantities of protein utilizing the principle of protein-dye binding. Anal. Biochem. 72: 248254.

19. Reinheimer $\mathrm{T}$, Bernedo $\mathrm{P}$, Klapproth $\mathrm{H}$, et al. (in press) Synthesis and storage of acetylcholine in isolated airways of rat, guinea-pig and man; species differences in the role of the airway mucosa. Am. J. Physiol.

20. Kozak M. (1986) Point mutations define a sequence flanking the AUG initiator codon that modulates translation by eukaroytic chromosomes. Cell 44: 283-292.

21. Marshall RD. (1972) Glycoproteins. Annu. Rev. Biochem. 41: 673-702.

22. Glass DB, el-Maghrabi MR, Pilkis SJ. (1986) Synthetic peptides corresponding to the site phosphorylated in 6-phosphofructo-2-kinase/ fructose-2, 6 bisphosphatase as substrates of cyclic nucleotide-dependent protein kinases. J. Biol. Chem. 261: 2987-2993.

23. Pinna LA. (1990) Casein kinase 2: an eminence grise in cellular regulation. Biochim. Biophys. Acta. 1054: 267-284.

24. Bourdon MA, Krusius T, Campbell S, Schwarz NB, Ruoshlati E. (1987) Identification and synthesis of a recognition signal for the attachment of glycosaminoglycans to proteins. Proc. Natl. Acad. Sci. U.S.A. 84: 3194-3198.

25. d'Souza SE, Ginsberg MH, Plow EF. (1991) Arginyl-glycyl-aspartic acid (RGD): A cell adhesion motifs. Trends Biochem. Sci. 16: 246-250.

26. Rajperts-de Meyts E, Heisterkamp N, Groffen J. (1988) Cloning and nucleotide sequence of human $\gamma$-glutamyl transpeptidase. Proc. Natl. Acad. Sci. U.S.A. 85: 8840-8844.

27. Nash B, Tate SS. (1984) In vitro translation and processing of rat kidney $\gamma$-glutamyl transpeptidase. J. Biol. Chem. 259: 678-685.

28. Finidori J, Laperche $Y$, Haguenauer-Tsapis R, Barouki R, Güellan G, Hanoune J. (1984) In vitro biosynthesis and membrane insertion of $\gamma$-glutamyl transpeptidase. J. Biol. Chem. 259: 4687-4690.

29. Laperche Y, Bulle F, Aissani T, et al. (1986) Molecular cloning and nucleotide sequence of rat kidney $\gamma$-glutamyl transpeptidase 
cDNA. Proc. Natl. Acad. Sci. U.S.A. 83: 937941.

30. Papandrikopoulou A, Frey A, Gassen $H$. (1989) Cloning and expression of $\gamma$-glutamyl transpeptidase from isolated porcine brain capillaries. Eur. J. Biochem. 183: 693698.

31. Suzuki H, Kumagai H, Echigo T, Tochikura T. (1989) DNA sequence of the Escherichia coli K-12 $\gamma$-glutamyl transpeptidase gene, ggt. J. Bacteriol. 171: 5169-5172.

32. Kyte AM, Doolittle F. (1982) A simple method for displaying the hydrophobic character of a protein. J. Mol. Biol. 157: 105-132.

33. Tate SS, Khadse V, Wellner D. (1988) Renal $\gamma$-glutamyl transpeptidases: Structural and immunological studies. Arch. Biochem. Biophys. 262: 397-408.

34. Wetmore LA, Gerard C, Drazen JM. (1993) Human lung expresses unique $\gamma$-glutamyl transpeptidases transcripts. Proc. Natl. Acad. Sci. U.S.A. 90: 7461-7465.

35. Matsuda Y, Tsuji A, Katunuma N. (1983) Studies on the structure of $\gamma$-glutamyl transpeptidase. III. Evidence that the amino terminus of the heavy subunit is the membrane binding segment. J. Biochem. (Tokyo) 93: 1427-1433.

36. von Heijne G. (1986) A new method for predicting signal sequences cleavage sites. Nucleic Acids Res. 14: 4683-4690.

37. Hussein AS, Walter RD. (1996) Purification and isolation of $\gamma$-glutamyl transpeptidase from Ascaris suum. Mol. Biochem. Parasitol. 77: 41-47.

38. Repetto Y, Letelier ME, Aldunate J, Morello A. (1987) The $\gamma$-glutamyl transpeptidase of Trypanosoma cruzi. Comp. Biochem. Physiol. 87B: 73-78.

39. Ikeda Y, Fujii J, Taniguchi N, Meister A. (1995) Human $\gamma$-glutamyl transpeptidase mutants involving conserved aspartates residues and the unique cysteine residue of the light subunit. J. Biol. Chem. 270: 1247112475.
40. Smith TK, Ikeda Y, Fujii J, Taniguchi N, Meister A. (1995) Different sites of acivicin binding and inactivation of $\gamma$-glutamyl transpeptidases. Proc. Natl. Acad. Sci. U.S.A. 92: 2360-2364.

41. Urban JF, Madden KB, Svetic A, et al. (1992) The importance of Th2 cytokines in protective immunity to nematodes. Immunol. Rev. 127: 205-220.

42. Kamata I, Yamada M, Uchikawa R, Matsuda S, Arizono N. (1995) Cysteine protease of the nematode Nippostrongylus brasiliensis preferentially evokes an IgE/IgGl antibody response in rats. Clin. Exp. Immunol. 102: 71-77.

43. Gardell SJ, Tate SS. (1979) Latent proteinase activity of $\gamma$-glutamyl transpeptidase. J. Biol. Chem. 254: 4942-4945.

44. Garraud O, Nkenfou C, Bradley JE, Perler FB, Nutman TB. (1995) Identification of recombinant filarial proteins capable of inducing polyclonal and antigen-specific IgE and IgG4 antibodies. J. Immunol. 155: 1316-1325.

45. van Ree R, Hoffman DR, van Dijk W, et al. (1995) Lol pXI, a new major grass pollen allergen, is a member of a family of soybean trypsin inhibitor-related proteins. J. Allergy Clin. Immunol. 95: 970-978.

46. Coyle AJ, Wagner K, Bertrand C, Tsuyuki S, Bews J, Heusser C. (1996) Central role of IgE in the induction of lung eosinophil infiltration and Th2 cytokine production: Inhibition by a non-anaphylactogenic anti-IgE antibody. J. Exp. Med. 183: 1303-1310.

47. Forman HJ, Skelton DC. (1990). Protection of alveolar macrophages from hyperoxia by $\gamma$-glutamyl transpeptidase. Am. J. Physiol. 259: L102-L107.

48. Kugelman A, Choy HA, Liu R, Ming Shi M, Gozal E, Forman HJ. (1995) $\gamma$-glutamyl transpeptidase is increased by oxidative stress in rat alveolar L2 epithelial cells. Am. J. Physiol. 11: 586-592.

Contributed by L. H. Miller. Accepted on September 4, 1996. 